\title{
Applications of Double Laplace Transform to Boundary Value Problems
}

\author{
Ranjit R. Dhunde ${ }^{1}$ and G. L. Waghmare ${ }^{2}$ \\ ${ }^{I}$ Department of Mathematics, Datta Meghe Institute of Engineering Technology \& Research, Wardha (MH-India) \\ ${ }^{2}$ Department of Mathematics, Institute of Science, Nagpur (MH-India)
}

\begin{abstract}
In this paper, we applied the method of Double Laplace Transform for solving the one dimensional Boundary Value Problems. Through this method the boundary value problem is solved without converting it into Ordinary Differential equation, therefore no need to find complete solution of Ordinary Differential equation. This is the biggest advantage of this method. The scheme is tested through some examples \& the results demonstrate reliability.

Mathematics Subject Classifications: 44Axx

Keywords: Boundary Value Problem, Double Laplace Transform, Inverse Laplace Transform, Partial derivatives.
\end{abstract}

\section{Introduction}

Integral transforms $[1,2]$ are extensively used in solving boundary value problems \& integral equations. The problem related to partial differential equation commonly can be solved by using a special Integral transform thus many authors solved the boundary value problems by using single Laplace Transform [6]. The Wave equation, Heat equation \& Laplace,s equations as three fundamental equations in mathematical Physics \& occur in many branches of Physics, in Applied mathematics as well as in Engineering. Eltayeb and Kilicman [3] has worked on the non-homogeneous wave equation with variable coefficients is solved by applying the Double Laplace Transform.

In 1990 [4 ], R. S. Dahiya \& Vinayagomorthy established several new theorems \& corollaries for calculating Laplace Transforms pairs of n-dimensions. They also considered two boundary value problems. The first was related to heat transfer for cooling off a very thin semi-infinite homogeneous plate into the surrounding medium solved by using double Laplace Transforms, the second, was heat equation for the semi-infinite slab where the sides of the slab are maintained at prescribed temperature.

Recently in 2013 [5], Aghili \& Motahhari have applied the Double Laplace Transform to solve second order Linear Differential equation with constant coefficients.

In this study, we use Double Laplace Transform to solve one dimensional boundary value problem, that is, Wave \& Heat equation. Henceforth the different problems of boundary value is solved without converting it into Ordinary Differential Equation, \& no need to find complete solution. So this method is very reliable \& convenient for solving boundary value problem.

The scheme is tested through three different examples which are being referred from [7, 8].

\section{Definition of double Laplace transform:}

First of all, we recall the following definitions given by Estrin \& Higgins [2].

Let $f(x, t)$ be a function of two variables $x$ and $t$, where $x, t>0$. The double Laplace transform of $f(x, t)$ is defined as

$$
L_{t} L_{x}\{f(x, t)\}=\bar{f}(p, s)=\int_{0}^{\infty} e^{-s t} \int_{0}^{\infty} e^{-p x} f(x, t) d x d t
$$

whenever the improper integral converges. Here $\mathrm{p}, \mathrm{s}$ are complex numbers.

\section{Existence of double Laplace transforms:}

Let $\mathrm{f}(\mathrm{x}, \mathrm{t})$ be a continuous function on the interval $[0, \infty)$ which is of exponential order, that is, for some $\mathrm{a}, \mathrm{b} \in R$.

Consider $\operatorname{Sup}_{x>0, t>0} \frac{|f(x, t)|}{e^{a x+b t}}<\infty$

In this case, the double Laplace transform of $f(x, t)$ that is

$$
L_{t} L_{x}\{f(x, t)\}=\bar{f}(p, s)=\int_{0}^{\infty} e^{-s t} \int_{0}^{\infty} e^{-p x} f(x, t) d x d t
$$

exists for all $p>a \& s>b \&$ is in fact infinitely differentiable with respect to $p>a \& s>b$. 
All functions in this study are assumed to be of exponential order.

\section{Double Laplace Transforms of Partial Derivatives:}

Double Laplace Transform for first partial derivatives with respect to $\mathrm{x}$ is defined as follows:

$$
\mathrm{L}_{\mathrm{t}} \mathrm{L}_{\mathrm{x}}\left\{f_{x}(x, t)\right\}=\mathrm{p} \bar{f}(\mathrm{p}, \mathrm{s})-\bar{f}(0, \mathrm{~s})
$$

Similarly, Double Laplace Transform for first partial derivatives with respect to $t$ is given by

$$
\mathrm{L}_{\mathrm{t}} \mathrm{L}_{\mathrm{x}}\left\{f_{t}(x, t)\right\}=\mathrm{s} \bar{f}(\mathrm{p}, \mathrm{s})-\bar{f}(\mathrm{p}, 0)
$$

Double Laplace Transform for second partial derivatives with respect to $\mathrm{x}$ is defined by

$$
\mathrm{L}_{\mathrm{t}} \mathrm{L}_{\mathrm{x}}\left\{f_{x x}(x, t)\right\}=p^{2} \bar{f}(\mathrm{p}, \mathrm{s})-p \bar{f}(0, \mathrm{~s})-\bar{f}_{x}(0, s)
$$

In a similar manner, Double Laplace Transform for second partial derivatives with respect to $t$ can be deduced from a single Laplace Transform

$$
\mathrm{L}_{\mathrm{t}} \mathrm{L}_{\mathrm{x}}\left\{f_{t t}(x, t)\right\}=s^{2} \bar{f}(\mathrm{p}, \mathrm{s})-s \bar{f}(\mathrm{p}, 0)-\bar{f}_{t}(p, 0)
$$

\section{Applications of Double Laplace Transform to Boundary Value Problem:}

Example 1: Solve the boundary value problem $\frac{\partial^{2} y}{\partial t^{2}}=\frac{\partial^{2} y}{\partial x^{2}}-\sin \pi x, 0<x<1$,

$t>0$ for

(i) $\quad \mathrm{y}\left(\mathrm{x}, 0^{+}\right)=0,0<x<1$

(ii) $\quad \mathrm{y}(0, \mathrm{t})=0, \mathrm{t}>0$

(iii) $\quad \mathrm{y}(1, \mathrm{t})=0, \mathrm{t}>0$

(iv) $\mathrm{y}_{\mathrm{t}}\left(\mathrm{x}, 0^{+}\right)=0,0<x<1$

Solution: Taking Double Laplace Transform

$$
\begin{aligned}
& s^{2} \bar{y}(p, s)-s \bar{y}(p, 0)-\bar{y}_{t}(p, 0)=p^{2} \bar{y}(p, s)-p \bar{y}(0, s)-\bar{y}_{x}(0, s)-\frac{\pi}{p^{2}+\pi^{2}} \frac{1}{s} \\
& \text { But } \bar{y}(p, 0)=0, \bar{y}(0, s)=0, \bar{y}_{x}(0, s)=0 \\
& \Rightarrow \quad s^{2} \bar{y}(p, s)=p^{2} \bar{y}(p, s)-\bar{y}_{x}(0, s)-\frac{\pi}{p^{2}+\pi^{2}} \frac{1}{s} \\
& \Rightarrow \quad \bar{y}(p, s)=\frac{1}{p^{2}-s^{2}} \bar{y}_{x}(0, s)-\frac{1}{p^{2}-s^{2}} \frac{\pi}{p^{2}+\pi^{2}} \frac{1}{s} \\
& \Rightarrow \quad \bar{y}(p, s)=\frac{1}{p^{2}-s^{2}} \bar{y}_{x}(0, s)-\frac{\pi}{\left(p^{2}+\pi^{2}\right)\left(s^{2}+\pi^{2}\right)} \frac{1}{s}+\frac{\pi}{\left(p^{2}-s^{2}\right)\left(s^{2}+\pi^{2}\right)} \frac{1}{s} \\
& \Rightarrow \quad \bar{y}(p, s)=\frac{1}{p^{2}-s^{2}}\left\{\bar{y}_{x}(0, s)+\frac{\pi}{\left(s^{2}+\pi^{2}\right)} \frac{1}{s}\right\}-\frac{\pi}{\left(p^{2}+\pi^{2}\right)\left(s^{2}+\pi^{2}\right)} \frac{1}{s} \\
& \Rightarrow \quad \bar{y}(p, s)=\frac{1}{2 s}\left\{\frac{1}{p-s}+\frac{1}{p+s}\right\}\left\{\bar{y}_{x}(0, s)+\frac{\pi}{\left(s^{2}+\pi^{2}\right)} \frac{1}{s}\right\}-\frac{\pi}{\left(p^{2}+\pi^{2}\right)\left(s^{2}+\pi^{2}\right)} \frac{1}{s} \\
& \text { Using } L_{x}{ }^{-1} \\
& \Rightarrow \quad \bar{y}(x, s)=\frac{1}{2 s}\left\{e^{s x}+e^{-s x}\right\}\left\{\bar{y}_{x}(0, s)+\frac{\pi}{\left(s^{2}+\pi^{2}\right)} \frac{1}{s}\right\}-\sin \pi x \frac{1}{s\left(s^{2}+\pi^{2}\right)} \\
& \text { As } x \rightarrow 1, \bar{y}(1, s) \rightarrow 0 \\
& \Rightarrow \quad \bar{y}(1, s)=\frac{1}{2 s}\left\{e^{s}+e^{-s}\right\}\left\{\bar{y}_{x}(0, s)+\frac{\pi}{\left(s^{2}+\pi^{2}\right)} \frac{1}{s}\right\}-\sin \pi \frac{1}{s\left(s^{2}+\pi^{2}\right)} \\
& \Rightarrow \quad 0=\frac{1}{2 s}\left\{e^{s}+e^{-s}\right\}\left\{\bar{y}_{x}(0, s)+\frac{\pi}{\left(s^{2}+\pi^{2}\right)} \frac{1}{s}\right\}-0 \\
& \Rightarrow \quad \bar{y}_{x}(0, s)=-\frac{\pi}{\left(s^{2}+\pi^{2}\right)} \frac{1}{s} \\
& \therefore(6) \Rightarrow \bar{y}(x, s)=-\sin \pi x \frac{1}{s\left(s^{2}+\pi^{2}\right)} \\
& \Rightarrow \bar{y}(x, s)=\sin \pi x \frac{1}{\pi^{2}}\left\{\frac{s}{s^{2}+\pi^{2}}-\frac{1}{s}\right\} \\
& \text { Using } L_{t}{ }^{-1} \text {, we get } \\
& \Rightarrow y(x, t)=(\sin \pi x) \frac{1}{\pi^{2}}\{\cos \pi t-1\}
\end{aligned}
$$

Example 2: A semi-infinite elastic beam is moving endwise with a velocity $-\mathrm{v}_{0}$ when one end is suddenly brought to rest, the other end remaining free. Then solve the resulting boundary value problem $y_{t t}(x, t)=$ $a^{2} y_{x x}(x, t), x>0, t>0$

$\mathrm{y}(\mathrm{x}, 0)=0, \mathrm{y}_{\mathrm{t}}(\mathrm{x}, 0)=-\mathrm{v}_{0}, \mathrm{y}(0, \mathrm{t})=0, \lim _{x \rightarrow \infty} y_{x}(x, t)=0$

Solution: Taking Double Laplace Transform

$$
\begin{gathered}
s^{2} \bar{y}(p, s)-s \bar{y}(p, 0)-\bar{y}_{t}(p, 0)=a^{2}\left\{p^{2} \bar{y}(p, s)-p \bar{y}(0, s)-\bar{y}_{x}(0, s)\right\} \\
\quad \operatorname{But} \bar{y}(p, 0)=0, \bar{y}(0, s)=0, \bar{y}_{t}(p, 0)=\frac{-v_{0}}{p} \\
\Rightarrow \quad s^{2} \bar{y}(p, s)+\frac{v_{0}}{p}=a^{2} p^{2} \bar{y}(p, s)-a^{2} \bar{y}_{x}(0, s) \\
\Rightarrow \quad \bar{y}(p, s)=\frac{a^{2}}{\left(a^{2} p^{2}-s^{2}\right)} \bar{y}_{x}(0, s)+\frac{v_{0}}{p\left(a^{2} p^{2}-s^{2}\right)}
\end{gathered}
$$


$\Rightarrow \quad \bar{y}(p, s)=\frac{a}{2 s}\left\{\frac{1}{p-\frac{s}{a}}-\frac{1}{p+\frac{s}{a}}\right\} \bar{y}_{x}(0, s)+\frac{v_{0}}{a^{2}} \frac{a^{2}}{2 s^{2}}\left\{\frac{1}{p-\frac{s}{a}}+\frac{1}{p+\frac{s}{a}}-\frac{2}{p}\right\}$

Using $L_{x}{ }^{-1}$

$\begin{array}{ll}\Rightarrow & \bar{y}(x, s)=\frac{a}{2 s}\left\{e^{\frac{s}{a} x}-e^{-\frac{s}{a} x}\right\} \bar{y}_{x}(0, s)+\frac{v_{0}}{2 s^{2}}\left\{e^{\frac{s}{a} x}+e^{-\frac{s}{a} x}-2\right\} \\ \Rightarrow & \bar{y}(x, s)=\left\{\frac{a}{2 s} \bar{y}_{x}(0, s)+\frac{v_{0}}{2 s^{2}}\right\} e^{\frac{s}{a} x}+\left\{\frac{v_{0}}{2 s^{2}}-\frac{a}{2 s} \bar{y}_{x}(0, s)\right\} e^{-\frac{s}{a} x}-\frac{v_{0}}{s^{2}} \\ \Rightarrow & \bar{y}_{x}(x, s)=\left\{\frac{a}{2 s} \bar{y}_{x}(0, s)+\frac{v_{0}}{2 s^{2}}\right\} e^{\frac{s}{a} x} \frac{s}{a}+\left\{\frac{v_{0}}{2 s^{2}}-\frac{a}{2 s} \bar{y}_{x}(0, s)\right\} e^{-\frac{s}{a} x}\left(\frac{-s}{a}\right)\end{array}$

Since $\lim _{x \rightarrow \infty} y_{x}(x, t)=0 \Rightarrow \lim _{x \rightarrow \infty} \bar{y}_{x}(x, s)=0$

$\therefore$ As $x \rightarrow \infty, \bar{y}_{x}(x, s)=0$

$\therefore(8) \Rightarrow 0=\frac{a}{2 s} \bar{y}_{x}(0, s)+\frac{v_{0}}{2 s^{2}}$

$\Rightarrow \bar{y}_{x}(0, s)=-\frac{v_{0}}{a s}$

$\therefore(7) \Rightarrow \quad \bar{y}(x, s)=\left\{\frac{v_{0}}{2 s^{2}}-\frac{a}{2 s}\left(-\frac{v_{0}}{a s}\right)\right\} e^{-\frac{s}{a} x}-\frac{v_{0}}{s^{2}}$

$\Rightarrow \quad \bar{y}(x, s)=\frac{v_{0}}{s^{2}} e^{-\frac{s}{a} x}-\frac{v_{0}}{s^{2}}$

Using $L_{t}{ }^{-1} \&$ by second shifting property, we get

$$
\begin{aligned}
& \Rightarrow \quad y(x, t)=v_{0}\left\{\left(t-\frac{x}{a}\right) u\left(t-\frac{x}{a}\right)-t\right\} \\
& \Rightarrow \quad y(x, t)= \begin{cases}v_{0}\left(-\frac{x}{a}\right), & \text { if } t>\frac{x}{a} \\
v_{0}(-t), & \text { if } t \leq \frac{x}{a}\end{cases}
\end{aligned}
$$

Example 3: A semi-infinite insulated bar which coincides with the $x$-axis, $x>0$, is initially at temperature zero. At $t=0$, a quantity of heat is instantaneously generated at the point $x=a$ where $a>0$. Find the temperature at any point of the bar at any time

$\mathrm{t}>0$.

Solution: The equation for heat conduction in the bar is

$\frac{\partial u}{\partial t}=k \frac{\partial^{2} u}{\partial x^{2}}, x>0, t>0$

The fact that a quantity of heat is instantaneously generated at the point $\mathrm{x}=\mathrm{a}$ can be represented by the boundary condition

$\mathrm{u}(\mathrm{a}, \mathrm{t})=\mathrm{Q} \delta(t)$

where $\mathrm{Q}$ is a constant $\& \delta(t)$ is the Dirac delta function. Also, since the initial temperature is zero \& since the temperature must be bounded, we have $\mathrm{u}(\mathrm{x}, 0)=0,|u(x, t)|<M$

Taking Double Laplace Transform on (9)

Since $\mathrm{u}(\mathrm{x}, 0)=0 \Rightarrow \bar{u}(p, 0)=0$

$$
s \bar{u}(p, s)-\bar{u}(p, 0)=k\left\{p^{2} \bar{u}(p, s)-p \bar{u}(0, s)-\bar{u}_{x}(0, s)\right\}
$$

$\Rightarrow s \bar{u}(p, s)=k\left\{p^{2} \bar{u}(p, s)-p \bar{u}(0, s)-\bar{u}_{x}(0, s)\right\}$

$\Rightarrow\left(k p^{2}-s\right) \bar{u}(p, s)=k p \bar{u}(0, s)+k \bar{u}_{x}(0, s)$

$\Rightarrow \bar{u}(p, s)=\frac{k p}{\left(k p^{2}-s\right)} \bar{u}(0, s)+\frac{k}{\left(k p^{2}-s\right)} \bar{u}_{x}(0, s)$

$\Rightarrow \bar{u}(p, s)=\frac{p}{\left(p+\sqrt{\frac{s}{k}}\right)\left(p-\sqrt{\frac{s}{k}}\right)} \bar{u}(0, s)+\frac{1}{\left(p+\sqrt{\frac{s}{k}}\right)\left(p-\sqrt{\frac{s}{k}}\right)} \bar{u}_{x}(0, s)$

$\Rightarrow \bar{u}(p, s)=\frac{1}{2}\left\{\frac{1}{p+\sqrt{\frac{s}{k}}}+\frac{1}{p-\sqrt{\frac{s}{k}}}\right\} \bar{u}(0, s)+\frac{1}{2} \sqrt{\frac{k}{s}}\left\{\frac{1}{p-\sqrt{\frac{s}{k}}}-\frac{1}{p+\sqrt{\frac{s}{k}}}\right\} \bar{u}_{x}(0, s)$

Using $L_{x}{ }^{-1}$

$\Rightarrow \bar{u}(x, s)=\frac{1}{2}\left\{e^{-\sqrt{\frac{s}{k}} x}+e^{\sqrt{\frac{s}{k}} x}\right\} \bar{u}(0, s)+\frac{1}{2} \sqrt{\frac{k}{s}}\left\{e^{\sqrt{\frac{s}{k}} x}-e^{-\sqrt{\frac{s}{k}} x}\right\} \bar{u}_{x}(0, s)$

$\Rightarrow \bar{u}(x, s)=\frac{1}{2}\left\{\bar{u}(0, s)-\sqrt{\frac{k}{s}} \bar{u}_{x}(0, s)\right\} e^{-\sqrt{\frac{s}{k}} x}+\frac{1}{2}\left\{\bar{u}(0, s)+\sqrt{\frac{k}{s}} \bar{u}_{x}(0, s)\right\} e^{\sqrt{\frac{s}{k}} x}$

Since $\mathrm{u}(\mathrm{x}, \mathrm{t})$ is bounded as $x \rightarrow \infty$.

$\therefore \bar{u}(x, s)$ is bounded as $x \rightarrow \infty$.

From boundedness condition, we require

$$
\begin{aligned}
& \frac{1}{2}\left\{\bar{u}(0, s)+\sqrt{\frac{k}{s}} \bar{u}_{x}(0, s)\right\}=0 \\
& \Rightarrow \bar{u}_{x}(0, s)=-\sqrt{\frac{s}{k}} \bar{u}(0, s)
\end{aligned}
$$


$\therefore(11) \Rightarrow \bar{u}(x, s)=\frac{1}{2}\left\{\bar{u}(0, s)+\sqrt{\frac{k}{s}} \sqrt{\frac{s}{k}} \bar{u}(0, s)\right\} e^{-\sqrt{\frac{s}{k}} x}$

$\Rightarrow \bar{u}(x, s)=\bar{u}(0, s) e^{-\sqrt{\frac{s}{k}} x}$

Since $\mathrm{u}(\mathrm{a}, \mathrm{t})=\mathrm{Q} \delta(t) \Rightarrow \bar{u}(a, s)=Q$

As $x \rightarrow a$ in $(12)$

$\therefore(12) \Rightarrow \bar{u}(a, s)=\bar{u}(0, s) e^{-\sqrt{\frac{s}{k}} a}$

$\Rightarrow Q=\bar{u}(0, s) e^{-\sqrt{\frac{s}{k}} a}$

$\Rightarrow \bar{u}(0, s)=Q e^{\sqrt{\frac{s}{k}} a}$

$\therefore(12) \Rightarrow \bar{u}(x, s)=Q e^{-(x-a) \sqrt{\frac{s}{k}}}$

Using $L_{t}{ }^{-1}$, we find the required temperature

$$
u(x, t)=\frac{Q}{2 \sqrt{\pi k t}} e^{\frac{-(x-a)^{2}}{4 k t}}
$$

The point source $\mathrm{x}=\mathrm{a}$ is some times called a heat source of strength $\mathrm{Q}$.

\section{References:}

[1] D. G. Duff, Transform Methods for solving Partial Differential Equations, Chapman and Hall/CRC, Boca Raton, F. L. 2004.

[2] A. Estrin \& T. J. Higgins, The Solution of Boundary Value Problems by Multiple Laplace Transformation, Journal of the Franklin Institute, 252 (2), 153 - 167, 1951.

[3] Adem Kilicman, Hassan Eltayeb, A note on defining singular integral as distribution and partial differential equations with convolution term, Elsevier, Mathematical \& Computer Modelling, 49(2013), 327-336.

[4] R. S. Dahiya, M. Vinayagamoorthy, Laplace Transform pairs of n- Dimensions \&Heat conduction problem, Mathl Comput. Modelling, Vol. 13, No. 10, pp. 35- 50, 1990.

[5] A. Aghili, A. Motahhari, Multi- Dimensional Laplace Transform for non- homogeneous Partial Differential Equations, Journal of Global research in Mathematical Archives, Vol. 1, No. 1, January 2013.

[6] H. Eltayeb \& A. Kilicman, A Note on Double Laplace Transform and Telegraphic Equations, Abstract \& Applied Analysis, Volume 2013.

[7] Joel L. Schiff, The Laplace Transform : Theory \& Applications, Springer, 1999.

[8] Murray R. Spiegel, Theory \& Problems of Laplace Transforms, Schaum's Outlines series, McGraw Hill, 1965.

[9] R. R. Dhunde, N. M. Bhondge \& P. R. Dhongle, Some Remarks on the Properties of Double Laplace Transforms, International Journal of Applied Physics \& Mathematics, Vol. 3, No. 4, July 2013, 293-295. 